\title{
Photocross-linked Anion Exchange Membranes with Improved Water Management and Conductivity
}

S. Piril Ertem ${ }^{1}$, Tsung-Han Tsai ${ }^{1}$, Melissa M. Donahue ${ }^{1}$, WenXu Zhang ${ }^{1}$, Ye Liu ${ }^{2}$, Soenke Seifert ${ }^{3}$, Andrew M. Herring ${ }^{2}$, E. Bryan Coughlin ${ }^{1 *}$

${ }^{1}$ Department of Polymer Science and Engineering, University of Massachusetts Amherst, 120 Governors Drive, Amherst, Massachusetts, 01003

${ }^{2}$ Department of Chemical and Biological Engineering, Colorado School of Mines, Golden, Colorado 80401

${ }^{3}$ X-Ray Science Division, Argonne National Laboratory, Argonne, Illinois 60439

Corresponding Author: E. Bryan Coughlin (Coughlin@mail.pse.umass.edu) 


\section{${ }^{1}$ H NMR characterization of the PI-ran-PVBCl copolymers}

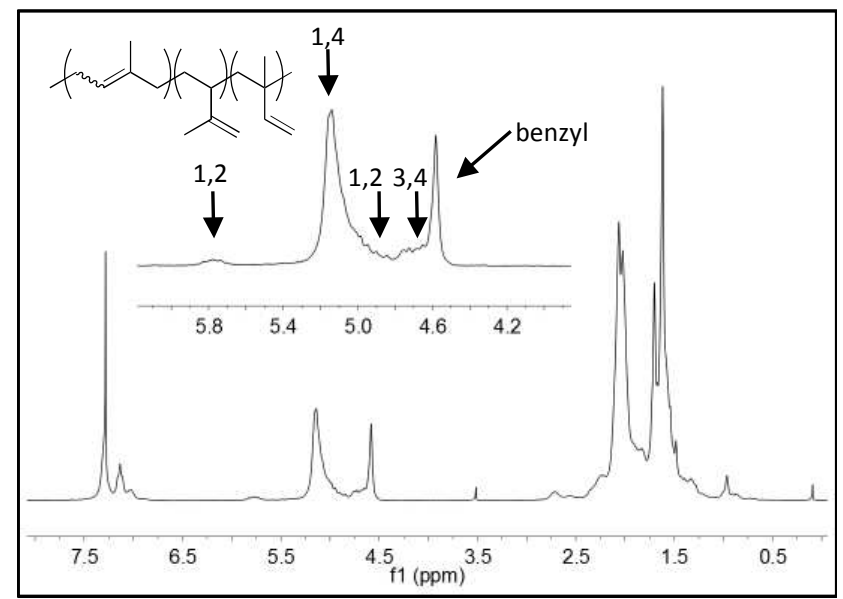

\section{Figure S1. ${ }^{1} \mathrm{H}$ NMR spectrum of PI-ran-PVBCl copolymer in $\mathrm{CDCl}_{3}$. Inset is enlarged vinyl proton region of isoprene with corresponding isomer assignments.}

Signals between $\delta$ 4.6-6.0 ppm are assigned to the vinyl protons of isoprene: The intense peak of the dominant isomer overlaps with the peaks of two geminal protons of 1,2isomer ( $\delta$ 4.8-5.0 ppm), while the signal for the vicinal proton of 1,2- isomer is observed around $\delta$ 5.0-5.3 ppm. The geminal protons of 3,4- addition give peaks around $\delta 4.6-4.8$ ppm.

\section{Control experiment for thiol-ene reaction efficiency with polyisoprene units}

A procedure similar to membrane fabrication procedure was used. Samples were prepared using PI-ran-PVBCl precursor with $72 \mathrm{~mol} \%$ isoprene with about 9\% 1,2- and 3,4-isoprene isomers. In duplicate, about $70 \mathrm{mg}$ polymer of polymer was dissolved in photoinitiator solution in THF $(0.6 \mathrm{~mL}, 5.8 \mathrm{mg} / \mathrm{mL})$. To solution 1 , one equivalent of 1 dodecanethiol $(138 \mu \mathrm{L})$ was added to the total amount of isoprene. To solution 2 , one equivalent of 1-dodecanethiol was added $(12 \mu \mathrm{L})$ to the total amount of 1,2- and 3,4- 
isomers. Films from each of the two solutions were drop cast onto Teflon ${ }^{\circledR}$ sheet, covered with a Petri dish and dried overnight in the dark. Dried films were exposed to UV light for 10 minutes ( $365 \mathrm{~nm}, 100 \mathrm{~mW} / \mathrm{cm}^{2}$ at working distance).

Figure S2 depicts the chemical shift region for isoprene for unreacted and reacted polymers. Benzyl protons were used as a reference to analyze the changes in vinylic proton signals. An overall decrease in double bond content was observed for both of the thiol-ene reacted systems. While the vicinal proton of 1,2- isomer could still be detected in both cases, it was greatly reduced compared to the unmodified polymer precursor. With a stoichiometric ratio of thiol to the total number of double bonds, nearly $50 \%$ of all 1,2- isomers were consumed. An overall reduction of the total double bond content was also observed (28\% at stoichiometric ratio), suggesting that the backbone double bonds of isoprene might also have reacted with thiols. Reactivity of the 1,4- isomer toward thiols has been shown for cross-linking of styrene- $b$-isoprene- $b$-styrene block copolymers with thiols. ${ }^{1}$ The persistence of vinylic peaks suggests that not all of the double bonds reacted, even at a stoichiometric ratio of thiol to isoprene. While it was not possible to detect the rate of reaction for individual isomers in these control experiments, it is reasonable to assume that sterically less hindered pendant double bonds would be consumed faster. 


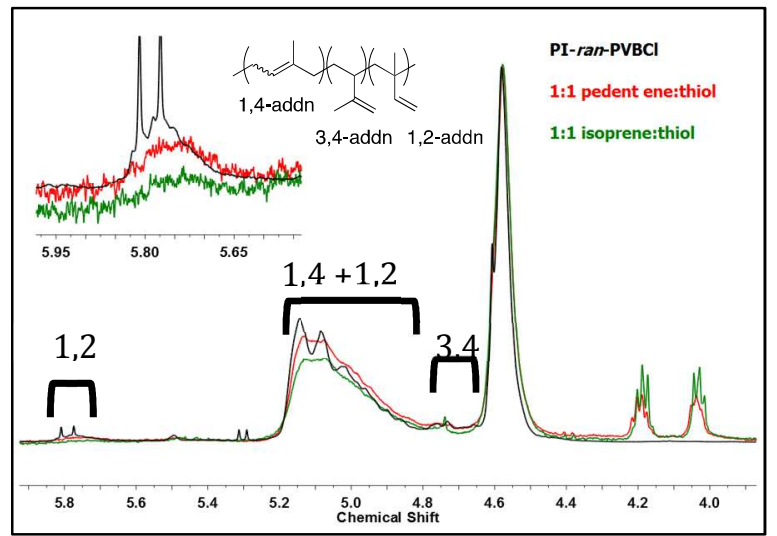

Figure S2. Thiol-ene reaction of isoprene isomers. Inset is expanded 1,2-isomer addition region between $\delta 5.9-5.6 \mathrm{ppm}$. Peaks between $\delta 4.3-3.9$ are due to photoinitiator residues.

\section{Sol-fraction Analysis}

A procedure similar to membrane fabrication was used. Membranes were prepared from PI-ran-P[VBTMA][Cl]-2.3 (77 mol\% isoprene with about 9\% 1,2- and 3,4-isoprene isomers) using 1,10-decanedithiol as the cross-linker. Three separate methanol solutions were prepared by adjusting the cross-linker ratio relative to the total amount of 1,2- and 3,4- isomers for each membrane as one, two, and three equivalents, respectively. Membranes were drop-cast onto a Teflon ${ }^{\circledR}$ surface, covered with a Petri dish for slow evaporation of solvent, and dried overnight in the dark. Dry membranes were crosslinked by exposure to UV light for 10 minutes $\left(365 \mathrm{~nm}, 100 \mathrm{~mW} / \mathrm{cm}^{2}\right.$ at working distance). Upon cross-linking, each membrane was dipped in $15 \mathrm{~mL}$ distilled water for 16 hours. A drop of water was transferred with a micro syringe onto ATR plate and water was allowed to evaporate. This method was repeated three times before collecting FTIR spectra of the water residue. 


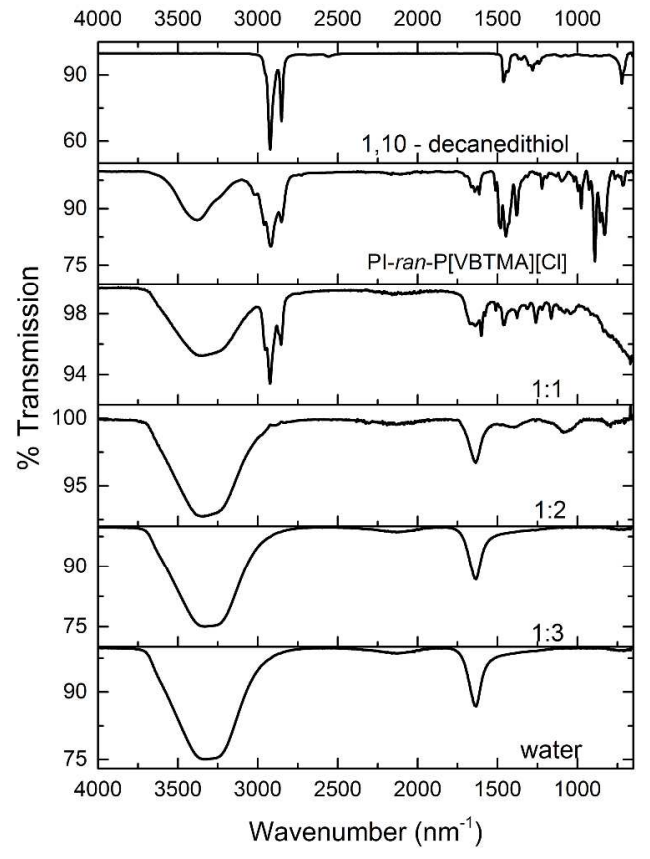

Figure S3. Sol-fraction FT-IR analysis of photocross-linked PI-ran-P[VBTMA][CI] copolymer with increasing ene to dithiol ratio.

\section{References}

(1) Decker, C.; Nguyen Thi Viet, T. J. Appl. Polym. Sci. 2000, 77, 1902-1912. 